\title{
Method to Correct Artifacts in Multilead ECG Using Signal Entropy
}

\author{
Beatriz Rodríguez-Alvarez ${ }^{1}$, José R. Ledea-Vargas ${ }^{1}$, Fernando E. Valdés-Pérez ${ }^{1}$, \\ Renato Peña-Cabrera ${ }^{2}$, and José-R. Malleuve-Palancar ${ }^{3}$ \\ ${ }^{1}$ Center for Neurosciences Studies, Images and Signals Processing (CENPIS), \\ Universidad de Oriente, Cuba \\ \{ledeav, bra, fvaldes\}@fie.uo.edu.cu \\ ${ }^{2}$ Biomedical Engineering Department, Universidad de Oriente, Cuba \\ renato.penalbio.fie.uo.edu \\ ${ }^{3}$ Cardiological Service, S. Lora Hospital, Santiago de Cuba, Cuba \\ malleuve@medired.scu.sld.cu
}

\begin{abstract}
Artifacts should be corrected previous heart rate variability analysis. A new method for artifact correction in multilead ECG is proposed in this paper. The method detects artifacts in the RR series, takes the corresponding segment of the multilead ECG, uses entropy of the signal for selecting the "cleanest" ECG channel, and uses the wavelet transform to recalculate positions of R peaks. The method was evaluated with ECG records of arrhythmia database MIT/BIH, with good results.
\end{abstract}

Keywords: ECG, artifact correction, entropy, wavelet, multilead ECG.

\section{$1 \quad$ Introduction}

The study of the electrocardiogram (ECG) provides great clinical information, not only for heart disease but others as neuropathy [1], ischemia [2], etc. Each beat is characterized by the points $\mathrm{P}, \mathrm{Q}, \mathrm{R}, \mathrm{S}$ and $\mathrm{T}$, providing information for diagnosis and prognosis. A topic widely studied is the heart rate variability (HRV) that provides information about the functioning of the heart and its interaction with the nervous system, in terms of the balance between sympathetic and vagal systems. HRV is described by statistical and spectral indexes calculated from time series obtained from the differences between $\mathrm{R}$ peaks (RR series) [3].

The study of HRV in long-term records of ECG obtained by ambulatory monitoring (usually 24 hours) is most reliable because the patient performs normal activities. This procedure allows studying the true behavior of the heart in certain situations. However, artifacts and interference contaminate the information in the HRV signal, so that many of the monitored patients can be wrongly classified as healthy subjects [1,3]. Modern devices allow recording multiple channels, but sometimes one of them may fail or contain artifacts that are not present in the other. Therefore researchers have implemented several methods and techniques in order to 
separate or extract useful information from the ECG signals, including the QRS complex detection, artifacts detection and correction, index calculations, etc.

The wavelet transform and entropy analysis have already been successfully used individually or combined in various areas of electrocardiology. In [4] a wavelet entropy (WS) based method is employed to detect QRS complexes in ECG signal from two significant channels. In addition, a method to detect multichannel ECG signals by using combined entropy is proposed in [5].

Methods proposed in [4] and [5] are very effective to detect QRS complexes in presence of artifacts such as baseline drift, and others. These artifacts are not present all the time in the signal, however the calculation based on wavelet and entropy is done for the whole signal. That means a high computing cost. The use of a lower cost computational method for detection of QRS complexes, only using the method based on wavelet and entropy in signal sections with artifacts seems to be a better option.

In this paper we propose a new method for correction of artifacts by combining the entropy for channel selection and the subsequent detection of $\mathrm{R}$ peaks with wavelet transform.

\section{Entropy and Wavelets}

\subsection{Entropy}

The basic concept of entropy has much to do with the uncertainty that exists in any experiment or random signal. It is also the amount of "noise" or "clutter" that contains or releases a system. Thus, one can speak about the amount of information carried by a signal.

ECG has varying amplitudes, both over time and between patients, or even with the measurement conditions; therefore it is desirable to provide methods for parameters analysis independent of signal amplitude. Entropy measures, which reflect the "order" of the signal, have this feature. The ECG signal has certain monotony from the viewpoint entropy. This can be seen as the appearance of regular and recurring waves (each heartbeat) with variations in the morphology and amplitude. Entropy measures, which quantify this order, look appropriate to detect events associated with muscle movements, noise, interference or artifacts in general [6].

To calculate the entropy is convenient to think about the histogram of the signal. This is done by making discrete the value range of the signal at $\mathrm{L}$ intervals. For each interval $i$ there will be a probability of occurrence $p_{i}$ given by:

$$
p_{i}=\frac{F_{i}}{\sum_{i=1}^{L} F_{i}}
$$

Where $F_{i}$ is the amount of signal values that are in the interval $i$. Shannon entropy $E$ of the signal $x$ is defined by: 


$$
E(x)=-\sum_{i=1}^{L} p_{i} \log _{2} p_{i}
$$

\subsection{Wavelets}

A wavelet expansion consists of translations and dilations of one fixed function, the wavelet $\psi \in L^{2}(R)$, where $R$ denotes the set of real numbers, and $L^{2}(R)$ denotes the set of measurable, square integrable one-dimensional functions [7]. In the continuous wavelet transform (CWT), the translation and dilation parameters can vary continuously. This means that we use the functions:

$$
\psi_{a, b}(t)=\frac{1}{\sqrt{|a|}} \psi\left(\frac{t-b}{a}\right) \quad \text { with a,b } \in \mathbf{R} \text { a } \neq \mathbf{0}
$$

Where the parameter $a$ is the scale and $b$ is the position parameter. The factor $|a|^{-1 / 2}$ has been introduced to guarantee energy preservation [8]. Given a continuous signal $f(t) \in L^{2}(R)$, the CWT of $f(t)$ is:

$$
W(a, b)=\int_{-\infty}^{\infty} f(t) \psi^{*}{ }_{a, b}(t) d t
$$

Where $\psi^{*}{ }_{a, b}(t)$ is the complex conjugate of $\psi_{a, b}(t)$. ECG signals are highly non stationeries, so the CWT is able to locate events in time and frequency. This analysis includes a windows technique with log time intervals for low frequency information, and short time intervals for high frequency information.

\section{Artifact Correction Algorithm}

The presented algorithm uses ECG signals in $n$ channels, using the redundancy of information being given. The first step is to create the series of positions of the QRS complexes ( $\mathrm{R}$ series) in any one of ECG channels. These QRS complexes are detected using a method based on the threshold of the derivative [9]. From R series, RR series are constructed in 300 beats segments, as recommended in [3] for short time RR series. The mean RR value of the series is calculated for each segment. The mean value is an artifact robust parameter of the series. Those points of the RR series that deviate more than $20 \%$ from the mean RR value are considered artifacts. This threshold has been evaluated empirically by the authors with good results. For each artifact, it is located in the ECG the heartbeat which originates the artifact, and sets the interval to be analyzed. To do this, choose a multi-channel ECG segment between the four seconds before the beat for the artifact detected and four seconds later. In the selected ECG interval the channel of smaller entropy is chosen, calculated according to (2) in the whole 8-second window. The R peaks are detected in this channel using 
the method based on the wavelet transform, reported in $[10,11]$, with the Gaussian Wavelet Daubechies function of fourth order and decomposition in 8 levels. The db4 contains good resemblance with the basic QRS shape of the ECG signal. This prominent feature of $\mathrm{db} 4$ insisted us to use it as the mother wavelet. The use of $8^{\text {th }}$ level Daubechies wavelets neutralizes the baseline drift.

Then $\mathrm{R}$ series is updated with these new values, the RR series is recalculated and the process is repeated until no artifact is present or if the number of iterations is greater than 20-25. This maximal number of iterations is a reasonable limit to the number of artifacts in a short time RR series. Figure 1 shows the steps of the algorithm.

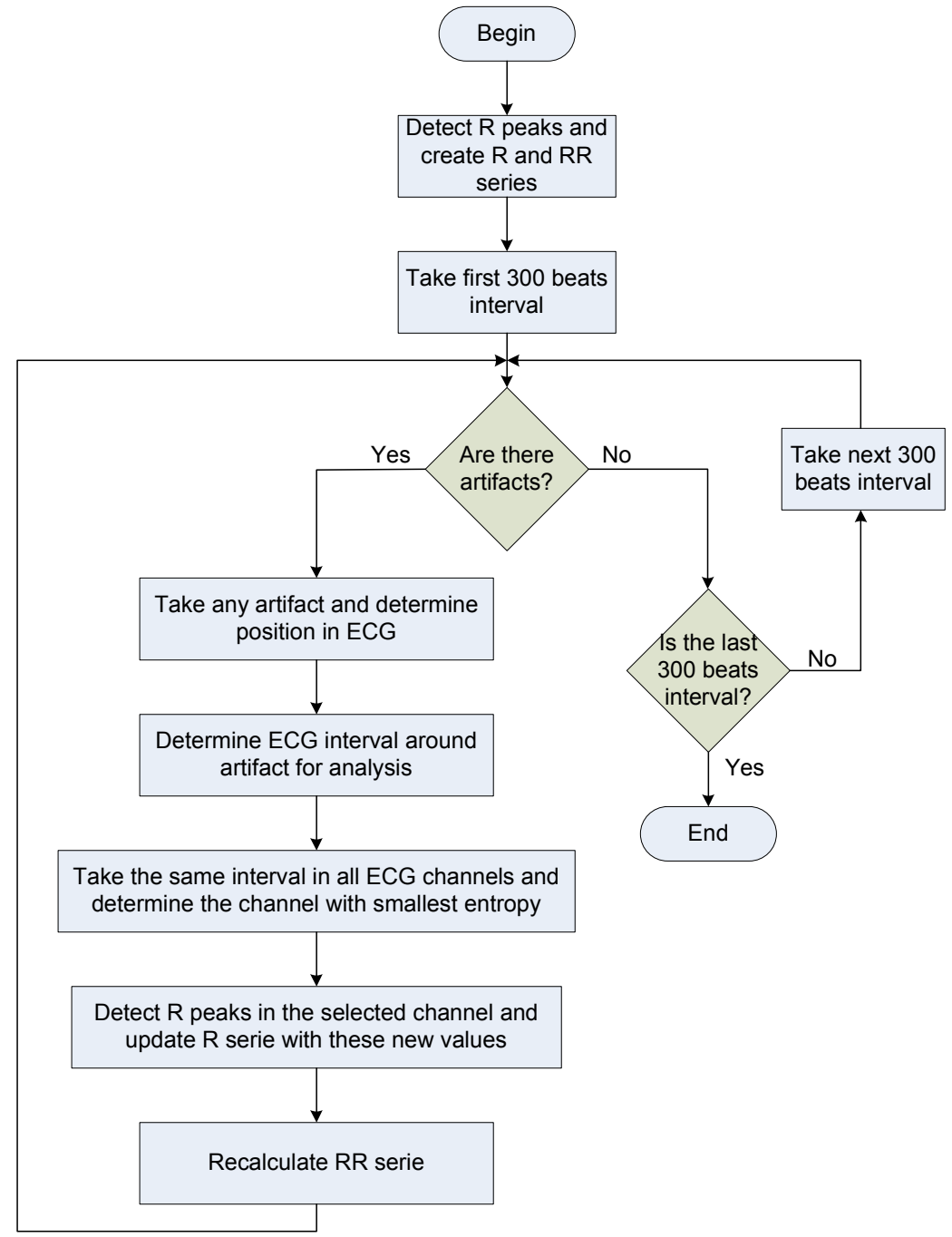

Fig. 1. Algorithm proposed for artifact correction 


\section{$4 \quad$ Results and Discussion}

The effectiveness of the proposed method was tested using ECG signals of arrhythmia database MIT/BIH. These ECG signals are 30 minutes registers in a range of 0-10 $\mathrm{mV}$, sampled at $360 \mathrm{~Hz}$. The resolution of each sample is 11 bits. The 48 records of this database have a variety of ECG morphologies, including heart disease, which makes them optimal for a qualitative and quantitative assessment of the algorithms described.

109270 beats (almost all of the base) were used, and the effectiveness of the proposed method to detect QRS complexes was proved. The results of this method were compared in two steps with the observations made by an expert. This is an indirect assessment of the effectiveness of the method to avoid the effect of the artifacts in the QRS detection in a multi-channel ECG.

The first step evaluates the effectiveness of the QRS detections by using $1^{\text {st }}$ derivative QRS detector applied in one channel. The second step makes artifact detection, correction with entropy and wavelet, and the evaluation of the whole method.

Three reference parameters for the analysis of the proposed algorithm were estimated: the sensitivity $(\mathrm{Se})$, positive predictability $\left(P^{+}\right)$and error $(E)$.

Sensitivity reflects the percentage of heartbeats which have been detected correctly by the algorithm on total existing heartbeat indicated by the expert. It shows the capacity of the detection method for detecting a peak. Sensitivity is calculated by:

$$
\operatorname{Se}(\%)=\frac{T P}{T P+F N} * 100
$$

Where:

TP: Number of true positives. This term represents the number of QRS complexes indicated by the expert and detected by the algorithm.

$F N$ : Number of false negatives. This term represents the number of QRS complexes indicated by the expert and not detected by the algorithm.

The value of $P^{+}$gives information about the percentage of beats correctly detected on total beats detected, ie, the probability that each point detected whether or not a peak $R$ and is determined by the following mathematical equation:

$$
P^{+}(\%)=\frac{T P}{T P+F P} * 100
$$

Where:

$F P$ : Number of false positives. It is determined by all the points identified as QRS complexes by the algorithm, and not indicated by the expert.

Another important aspect to evaluate each one of the described algorithms is the error, given by the following mathematical expression:

$$
E(\%)=\frac{F P+F N}{T L} * 100
$$

Where $T L$ is the total number of analyzed beats $(T P+F P)$. 
Tables 1 and 2 show the results of applying the steps of the method to the MIT$\mathrm{BIH}$ database. Table 1 indicates large number of FN and FP when the 1st derivative QRS detector is applied. Table 2 shows how the number of FN and FP strongly decrease when the artifacts are detected and corrected by the entropy and wavelet method.

Table 1. The results of the first step

\begin{tabular}{|c|c|c|c|c|c|c|}
\hline $\mathbf{T L}$ & $\mathbf{T P}$ & $\mathbf{F N}$ & $\mathbf{F P}$ & $\boldsymbol{S e}(\boldsymbol{\%})$ & $\mathbf{P}^{+}(\boldsymbol{\%})$ & $\mathbf{E}(\boldsymbol{\%})$ \\
\hline 109270 & 101458 & 7812 & 1723 & 92.85 & 98.33 & 8.73 \\
\hline
\end{tabular}

Table 2. The results of the proposed algorithm

\begin{tabular}{|c|c|c|c|c|c|c|}
\hline $\mathbf{T L}$ & $\mathbf{T P}$ & $\mathbf{F N}$ & $\mathbf{F P}$ & $\boldsymbol{S e}(\boldsymbol{\%})$ & $\mathbf{P}^{+}(\boldsymbol{\%})$ & $\mathbf{E}(\boldsymbol{\%})$ \\
\hline 109270 & 108717 & 553 & 120 & 99.49 & 99.89 & 0.67 \\
\hline
\end{tabular}

Table 2 illustrates that the proposed method achieves an average sensitivity of $99.49 \%$, the positive prediction of $99.89 \%$ and the failed detection of $0.67 \%$. These results show high values of sensibility and positive prediction, besides a very low error rate. Then it is possible to conclude that the proposed method can be applied with good results in the detection of QRS complexes in multilead ECG with artifacts. This result may be useful in studies of HRV, and in other applications that require high precision and dependability of the results. The method shows difficulties when artifacts appear in all the channels at the same time. This is not very frequent unless it is an artifact of physiologic origin.

\section{Conclusions}

The method developed shows very good indicators in detecting QRS complexes in multilead ECG with artifacts, based on the use of the entropy for the selection of the channel, together with the detection of devices using the transformed of wavelet.

\section{References}

1. García González, M.A.: Estudio de la variabilidad del ritmo cardíaco mediante técnicas estadísticas, espectrales y no lineales. Tesis doctoral para la obtención del título de doctor. Departamento de Ingeniería Electrónica. Universidad Politécnica de Cataluña (1998)

2. Lemire, D., Pharand, C., Rajaonah, J.-C., Dubé, B., LeBlanc, A.-R.: Wavelet Time Entropy, T wave morphology and myocardial ischemia. IEEE Transactions in Biomedical Engineering 47(7), 967-970 (2000) 
3. Malik, M., et al.: Heart rate variability. Standards of measurement, physiological interpretation, and clinical use. Task Force of The European Society of Cardiology and The North American Society of Pacing and Electrophysiology. European Heart. Journal 17, 354-381 (1996)

4. Boqiang, H, Yuanyuan, W: Detecting QRS Complexes of Two-channel ECG Signals by Using Combined Wavelet Entropy. In: IEEE 3rd International Conference on Bioinformatics and Biomedical Engineering, ICBBE 2009, June 11-13, pp. 1-4 (2009) E-ISBN: 978-14244-2902-8a

5. Mehta, S.S., Lingayat, N.S.: Combined entropy based method for detection of QRS complexes in 12-lead electrocardiogram using SVM. Computers in Biology and Medicine 38, 138-145 (2008)

6. Bermúdez, A.N., Spinelli, E.M., Muravchik, C.M.: Detección de eventos en señales de EEG mediante Entropía Espectral. XVIII Congreso Argentino de Bioingeniería SABI 2011 - VII Jornadas de Ingeniería Clínica, Mar del Plata, 28 al 30 de Septiembre (2011)

7. Chui, C.K.: Wavelets: a tutorial in theory and applications. Academic Press (1992)

8. Mallat, S.: A theory for multiresolution signal decomposition: the wavelet representation. IEEE Pattern Anal. and Machine Intell. 11(7), 674-693 (1989)

9. Köhler, B., Hennig, C., Orglmeister, R.: The Principles of Software QRS Detection. IEEE Engineering in Medicine and Biology, 42-57 (February 2002)

10. Mahmoodabadi, S.Z., Ahmadian, A., Abolhasani, M.D.: ECG feature extraction using daubechies wavelets. In: Proceedings of the Fifth IASTED International Conference Visualization, Imaging and Image Processing, Benidorn, Spain, September 7-9, pp. 343-348 (2005)

11. Vera, O.E., Duque-Cardona, E., Rivera-Piedrahita, J.: Extracción de características de la señal electrocardiográfica mediante software de análisis matemático. Scientia Et Technica, Universidad Tecnológica de Pereira, Colombia, vol. XII(31), pp. 59-64 (Agosto 2006) 